\title{
Application of the Ontology Concept for the Needs of Theoretical Mechanics
}

\author{
Aivars Vilkaste, Peter Grabusts \\ Rezekne Higher Education Institution, Faculty of Engineering. \\ Address: Atbrivosanas aleja 115, Rezekne, LV-4601, Latvia
}

\begin{abstract}
The concepts of theoretical mechanics have been sufficiently well studied, they are used both for educational purposes and practical application. Nowadays, the use of ontologies is developing rapidly, thus allowing acquiring knowledge about the specific field of application. The author of this research analyses appropriateness of ontologies for the needs of theoretical mechanics. The study shows the use of graphs in the work with taxonomy concepts and describes the key notions of the ontologies. The author has tried to develop the concept of domain ontology for better understanding of the key notions of theoretical mechanics with a help of Protégé, which would be useful for students in the process of studying theoretical mechanics.
\end{abstract}

Keywords: ontology, Protégé, taxonomy, theoretical mechanics.

\section{INTRODUCTION}

One of the many engineering activities is the research of material objects, i.e. any physical matter that has some mass. Given that all material objects interact with each other and due to this effect they can move in relation to each other, it can be concluded that there are different systems of material objects whose existence and development can be modulated depending on the environment in which the system is functioning or depending on the impact of other systems, individual objects or forces on this system. Theoretical mechanics studies material objects, it explores mechanical movements and mutual positions of material objects and their positions of equilibrium. Theoretical mechanics sees material objects as material points, absolutely solid bodies or their combinations in mechanical system with a certain weight, despite the fact that they differ in shape, size, material and mass. Thus, the object of the study is an abstract model instead of a real physical formation. Theoretical mechanics forms a common theoretical basis for all the technical objects, it is closely related and is developing together with them. Resistance of materials, theory of elasticity, fluctuation theory, machinery and mechanical theory, structural mechanics, theory of plasticity, hydrodynamics, technological machinery theory of various fields and many other theories are based on the general rules of theoretical mechanics. Theoretical mechanics is a basis for general technical preparedness which allows analyzing and evaluating operation of different systems and use of the obtained results, in order to solve various technical problems and tasks.
The studied material objects and systems of these objects are interconnected and interdependent. Interaction of material objects and object-systems is usually very diverse and complex, so there is a need to structure and display a wide range of various information, in order to ensure a common understanding of the studied objects and their systems, as well as to find rational and effective solutions for specific problems or tasks.

\section{SOME NEEDS OF THEORETICAL MECHANICS}

Theoretical mechanics studies mechanical motions of material objects and their positions of equilibrium, as well as reciprocal mechanic impact of material objects.

The following aspects should be taken into account:

1. Object of study is a material point (absolutely solid body with a constant distance between any two points thereof).

2. Mechanical system is a set of any material points that mechanically interact with each other.

3. Mutual interaction of material objects and mechanical systems is characterized by the physical concept of force $\vec{F}$, which is the cause of the speed, direction of a motion or deformation.

4. Material objects and mechanical systems are affected by external forces $\vec{F}_{e}$ and internal forces $\vec{F}_{i}$. 
5. Depending on the material object or mechanical system, the same force may be both external and internal.

6. System of forces is formed by several forces that affect the same material object.

It should be also taken into account that any material body in mechanics is seen as a mechanical system consisting of a set of material points. Besides, an absolutely solid body is considered to be a constant mechanical system because the distance between its points always remains the same.

There are various forces in natural environment: Ampere force, Archimedean force, repulsive force, external forces, friction force, lift force, elasticity force, gravitational force, internal forces, Coulomb force, motion stimulating force, motion refraining force, total force, Lorentz force, interaction force, molecular forces, attractive force, repulsive force, reaction force, spring force, gravity, weight, impact force, hauling force, etc. (see Fig. 1) [1, 3].

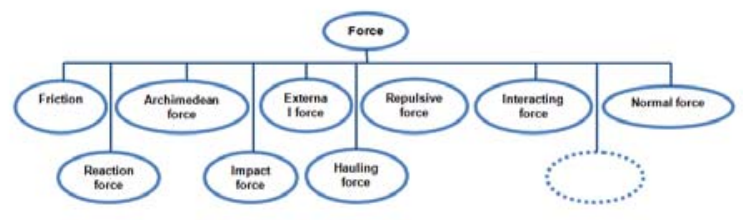

Fig.1. Forces

Besides, the study covers fundamental and nonfundamental forces, forces of direct and indirect impact, as well as conservative and non-conservative forces, etc. The existing forces have both common and different characteristics and can be grouped by selecting different features, for example grouping according to their performance (see Fig. 2). Some of the forces are studied in theoretical mechanics.

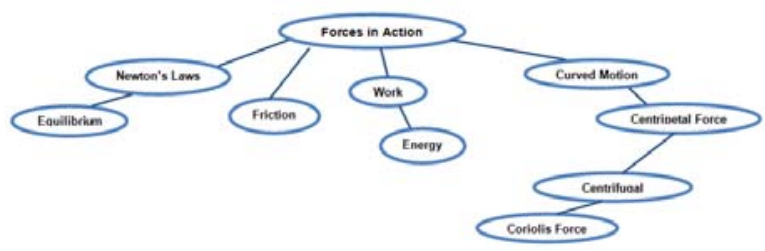

Fig. 2. Distribution of forces by their performance

It can be seen, that when addressing the tasks of theoretical mechanics:

- it is important to accurately determine the type of the force and its compliance with the mechanical action;

- to anticipate the effects of the impact of the force or the system of forces on the material point or mechanical system.

Henceforth, the authors shall explore the needs of the theoretical mechanics by using ontology.

\section{POTENTIAL OF ONTOLOGIES}

Over the past twenty years, the term "ontology" has been introduced and is commonly used in the engineering science, it is used to describe the models with different levels of detail (structuring) in an understandable way, as well as to demonstrate wide and complex information through conceptual schemes. Ontologies allow divided usage of unified understanding of information structure, reuse of the knowledge of problem domain, making clear assumptions in a specific problem domain, separation of knowledge of problem domain from operational knowledge, as well as analysis of the knowledge of problem domain.

The term "ontology" comes from philosophy. In philosophy, ontology is a study of the nature of being ("ontos" - being, "logos" - study), defining characteristics of the existence, where general basics, principles, structure and regularities are described. Each discipline has its own ontology, assumptions about what things exist or may exist in reality, what could be the conditions of their existence, and what they might be dependent on.

Originally, the term "ontology" was introduced in some areas of the research of artificial intelligence: knowledge engineering, natural language processing and knowledge representation. In late 1990's, the term "ontology" became widespread in other areas: intelligent information integration, information retrieval from the Internet, knowledge management, and e-commerce [11]. Ontology is also widely used in semantic webs.

In 1991, R. Neches and his colleges were one of the first to define ontology that is being used in the IT field and may be used in engineering science as well: "An ontology defines the basic terms and relations comprising the vocabulary of a topic area as well as the rules for combining terms and relations to define extensions to the vocabulary" [12].

This definition shows the composition of ontologies and provides some guidelines for building them: identification of basic terms and relations, identification rules for combining terms, provision of definition of terms and relations. Basing on this definition of ontology, it can be concluded that ontology contains not only ontology terms that are precisely defined, but also the knowledge that can be derived from them. T. R. Gruber defines the ontology in the following way: "An ontology is a formal explicit specification of a shared conceptualization" [7]. Ontologies are often equated with taxonomic hierarchies of classes. Thus, the aim of ontologies is to accumulate knowledge in a general and formal way.

Literature sources provide more than ten different definitions of ontology. Although each author offers his definition of ontology, they are all based on the T. R. Gruber's definition of ontology, which is modified or updated. 
Ontologies are often interrelated and are used to create knowledge bases, because "an ontology is a hierarchically structured set of terms for describing a problem domain that can be used as a skeletal foundation for a knowledge base" [11]. V. Devidzic states that "an ontology is a set of terms and relations where all concepts are defined and interpreted in a declarative way" [9]. M. Uschold and R. Jasper define that "an ontology may take a variety of forms, but necessarily it will include a vocabulary of terms, and some specification of their meaning. This includes definitions and an indication of how concepts are inter-related which collectively impose a structure on the domain and constrain the possible interpretations of terms" [15].

When analyzing definitions of ontologies, N. Guarino and other scientists [13] note than an ontology should be "human-understandable and machine-readable", as well as "a shared view on conceptualization of the problem domain".

Ontologies can be classified in different forms. One of the most popular forms of classification is the one proposed by Guarino, who classified types of ontologies according to their level of dependence on a particular task or point of view [6]:

- Top-level ontologies: describe general concepts like space, time, event, which are independent of a particular problem or domain.

- Domain-ontologies: describe the vocabulary related to a generic domain by specializing the concepts introduced in the top-level ontology.

- Task ontologies: describe the vocabulary related to a generic task or activity by specializing the top-level ontologies.

- Application ontologies: they are the most specific ones. Concepts often correspond to roles played by domain entities. They have a limited reusability as they depend on the particular scope and requirements of a specific application.

V. Solovyov, B. Dobrov, V. Ivanov and V. Lukasevic have formulated basic characteristics of ontologies [4]:

- at logical level, each ontology complies with a specific theory, but sometimes there is a specific fixed model;

- ontologies are created basing on the module principle: when identifying a new ontology, the previous ontologies can be used;

- ontologies have to be easily understandable and usable.

B. Dobrov and V. Lukashevic have found a formal designation for ontologies [4]:

$$
O=\{X, R, F\}
$$

where $O$ - stands for ontology of domain, $X-$ a set of domain concepts, $R$ - a set of relations of these concepts, $F-$ a set of function interpretations of concepts and relations between them.

V. Graudina in her article on ontologies [11] has analyzed 12 different ontology classification schemes and has elaborated a scheme classification. Ontologies may be classified in accordance with their expressiveness, weight, degree of formalization, or in line with the generality of ontologies, generality of the studied problem, creation process and the reflected worldviews. Taking into account the classification by the generality of ontology, we can talk about ontology classification by dependence on a specific application or task, by asking ontological knowledge of languages, possibilities of knowledge visualization and other parameters. One of the types of ontologies are application ontologies that depend on the specific application, objectives and tasks, as well as the methods for their solution, they contain the knowledge needed for the specific applications. Application ontologies describe the concepts that depend on the task ontology and on the domain ontology.

Methods and methodology for the creation of ontologies are studied by the ontology engineering. It describes the set of designing principles, development processes and activities, as well as supports technology and systematic methodologies, which in turn facilitate the development and use of the complete cycle of ontologies, i.e. designing, implementation, evaluation, validation, maintenance, distribution, mapping, integration, sharing and reuse. Ontology engineering aims to make explicit the knowledge and procedures contained in applications. It offers guidelines for solving interoperability problems caused by semantic barriers. Ontology engineering provides [10]:

1) Determination of the concept class in the ontology.

2) Implementation of taxonomy in classes (subclass - class - superclass).

3) Elaboration of a structure of concepts and situations.

4) Determination of characteristics and the value of these characteristics.

5) Procedures for conclusions and situation transformation.

N. F. Noy and D. L. McGuinness [8] offer seven steps for creation of ontologies, however, they do not consider that this is the only correct method for creation of ontologies. Ontology building process is iterative, modification and supplementation take place throughout the whole building process. However, before the creation of an ontology, the author suggests to answer some basic questions:

1) What is the domain the ontology will cover?

2) What are the aims of using the ontology?

3) What are the types of questions that should be answered with a help of the ontology?

4) Who will use and maintain the ontology? 
The answers to these questions may change during the ontology designing process, but they always help limiting the scope of the model.

Step 1. Determine the domain and scope of the ontology.

Step 2. Consider reusing existing ontologies.

Step 3. Enumerate important terms in the ontology.

Step 4. Define the classes and the class hierarchy.

Step 5. Define the properties of classes - slots.

Step 6. Define the facets of the slots.

Step 7. Create instances.

Taking into account the definition, classification of ontologies and their building methodology, it can be stated that the concept of ontology may be applied in order to study the mechanical movements of material objects and their positions of equilibrium, as well as reciprocal position of material objects, which is the task of theoretical mechanics.

Thus, an ontology is an explicit representation of knowledge. It is a formal, explicit specification of shared conceptualizations, representing the concepts and their relations that are relevant for a given domain of discourse [7].

Ontologies are widely applied in the Semantic Web, but there is no information about the use of ontologies in theoretical mechanics.

Considering the fact that the ontology should be "human-understandable and machine-readable" [6], as well as "easy understandable and usable" [4], the author shall show the ontology building process in two ways. Firstly, the use of graphs and graph theory, because information that is correctly visually encoded is more easy to understand and process than any other type of information. Secondly, use of the open-source platform Protégé [14], which allows building models of knowledge in a given field.

\section{USE OF GRAPHS IN TAXONOMY DEFINING}

Graph is a system that consists of a finite number of objects of two types (vertices and edges), and each edge is connected with two vertices. Graph may be drawn by depicting its verticals with nodes, while each edge is depicted by an arbitrary line connecting the relevant vertices (see Fig. 3).

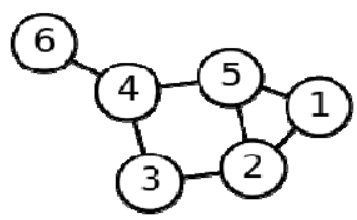

Fig. 3. Graph with 6 vertices and 7 edges

Counting and implementation of graph models make the analysis and solution of tasks demonstrative and effective [2], which is important when building application ontology. Visualization of relations by graph helps analyzing them both theoretically and experimentally, by using information technologies [2].

It should be noted that the graph concept can be generalized in the following way: subsets of the set of graph edges are taken as graph edges, besides these subsets (edges) can reoccur. In this case, we obtain a hypergraph that is an incidence system of objects (hypergraph vertices) and a finite system consisting of non-empty sets of these objects (faces or edges of the hypergraph) (see Fig. 4).
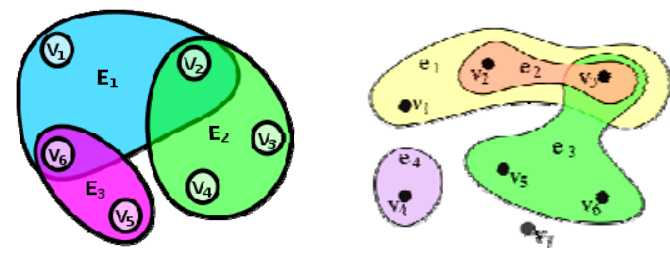

Fig. 4. Hypergraph models

Mathematical description of a graph is the following: graph is a pair of sets $G=(V ; E)$, where elements of the set $\mathrm{V}$ are vertices $V=\left\{v_{i}\right\}, i \in I=$ $\{1,2, \ldots, n\}$, while elements of the set $E$ are edges that connect the vertices $\mathrm{E}=\left\{e_{j}\right\}, j \in J=\{1,2, \ldots, m\}$; each edge is a subset of the set of vertices V. $E \subset$ $V \times V$, where $V \times V$ is Descartes multiplication of sets and each edge is a pair $\left(v_{1}, v_{2}\right)$ or $\left(v_{2}, v_{1}\right)$. Definition of hypergraph is similar: hypergraph is a pair of sets $H=(V ; E)$, where $V \neq\{\varnothing\}$ and $E \subseteq$ $P(V)$. Edges of the hypergraph (elements of the set E) correspond to arbitrary subsets of the set $\mathrm{V}$ of vertices.

The graph theory defines orientated and nonorientated hypergraphs and, traditionally, both vertices and edges are colored in a non-orientated graph [2]; it assigns positive integers to vertices or edges or colors vertices and edges of the graph. This principle may be applied to hypergraphs too, as it is a generalization of the graph concept. A colored, partly-orientated hypergraph is called semantic hypergraph that shall be used for ontology building. The above mentioned facts allow defining ontology as a semantic hypergraph:

$$
H=O(X, R, I)
$$

where $\mathrm{X}$ is a set of concepts (vertices), $\mathrm{R}-\mathrm{a}$ set of relations (edges), and $\mathrm{I}-\mathrm{a}$ set of predicates that define the incidence (connectivity) in the hypergraph.

Thus, the model of graph theory may be used for demonstrative implementation of taxonomy in classes (subclass - class - superclass).

\section{$\mathrm{V}$ POSSIBILITIES OF ONTOLOGY IN THE PROCESS OF DEFINING THE CONCEPTS OF THEORETICAL MECHANICS}

When addressing the problems of theoretical mechanics, it is important to determine the type of the force and its compliance with the mechanical action. 
In view of this, the author shall demonstrate a fragment of the ontology by using a model of graph theory.

When creating an ontology of forces, all existing forces may be seen as its elements, however it should be taken into account that the theoretical mechanics studies interaction of material objects and mechanical systems. Then, when creating the ontology, the author shall demonstrate the direct and indirect forces. There shall be two subclasses ("Contact forces" and "Action - at - a - Distance Forces") in the class named "Forces". The author shall demonstrate the relations between the concept classes, subclasses and their elements in a form of organized taxonomy. For the needs of taxonomy, the relations $A$ kind of shall be used. The fragment of taxonomy for the ontology "Force" can be seen in the Figure 5.

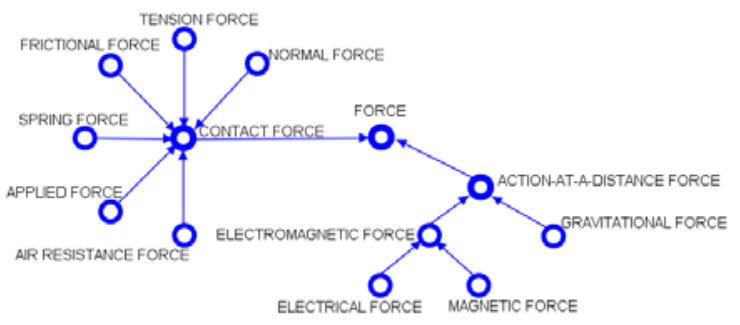

Fig. 5. Taxonomy fragment

It should be noted that one object may contain a part of another object. For example, material object is a part of mechanical system, which can also be seen as a material object, the weight of the material object is part of the friction force. This type of relations is named Part_of, and it is sufficient for such concepts to list their components. In many cases, it is important to show the relations between the components. In such cases, the structure concepts are often used and the relations Have_structure are applied to them. One of the elements of the subclass Contact forces is Frictional Force, whose structure may be demonstrated in a form of semantic web by using a hypergraph (see Fig. 6).

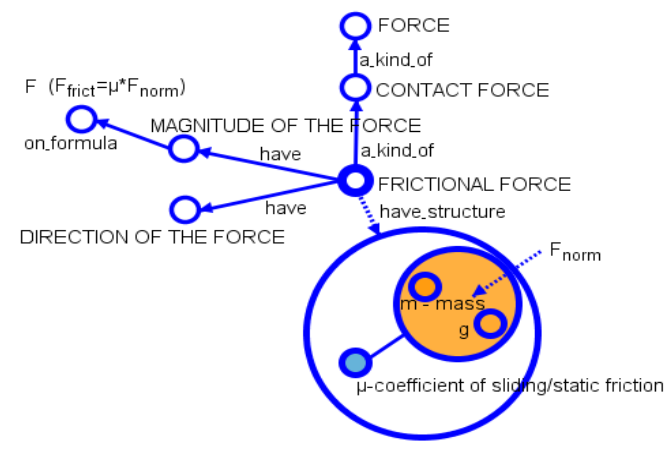

Fig. 6. The concept of Frictional Force structure fragment
It can be seen that the model of graph theory used for the study makes the information needed for the theoretical mechanics of ontologies easily perceivable and understandable.

Basing on the assumptions about the needs of tasks of theoretical mechanics, it would be useful to develop a general approach to the framework that could be helpful for this type of task class (see Fig. 7) [5].

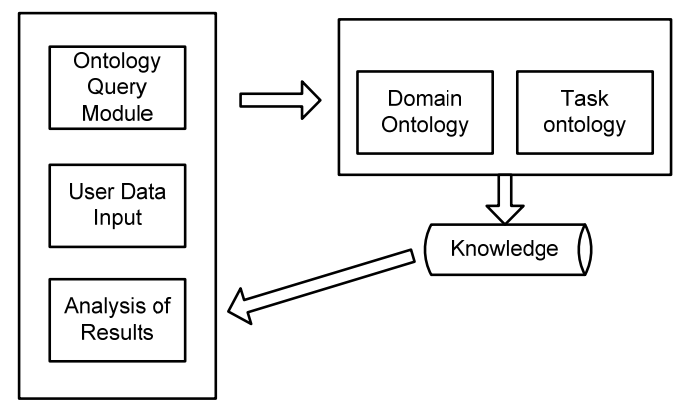

Fig. 7. Prototype of ontology-based framework for the needs of tasks of theoretical mechanics

Developing framework Protégé OWL tool is used for construction of this prototype [14]. Protégé is a special tool, which is thought to create and edit ontology, but OWL (Web Ontology Language) is a language through which it is possible to define the ontology. OWL ontology may include descriptions of classes, their characteristics and their instances. OWL formal semantics describes how, by using these data, to get information which was not openly described in ontology, but which is derived from the data semantics.

Protégé is a free, open source ontology editor and a knowledge acquisition system. Protégé provides a graphic user interface to define ontologies. In Protégé environment a number of knowledge-modeling structures and actions that support ontology creation, visualization and editing of different display formats are implemented. It also includes deductive classifiers to validate that models are consistent and to infer new information based on the analysis of ontology.

Protégé is an extensible knowledge model. Protégé's primitive - the component of its knowledge model - provide classes, instances of these classes, frame representing attributes of classes and instances.

Ontology development with the help of Protégé starts with the definition and description of classes hierarchy, and then instances of these classes and different types of relationships (properties in Protégé) in order to put more meaningful information within the ontology are assigned [5].

Looking at the possibilities of applying ontologies for the needs of theoretical mechanics, at the beginning of the research the author studied only the domain ontology. In order to demonstrate it, similarly to using the model of graph theory, the class "Forces" was chosen (see Fig. 8 and Fig. 9). 


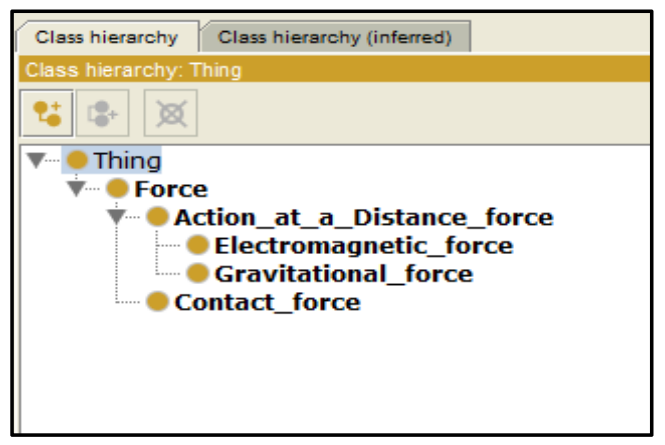

Fig. 8. Subclasses of the class „Force” in the „Class hierarchy” tab of Protege

It is assumed that a class "Force" has been introduced and that this class contains two subclasses Contact force and Action - at-a-Distance force.

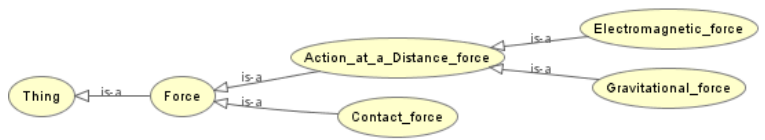

Fig. 9. Visualization of subclasses of the class Force in OWLViz tab of Protégé

The subclass Contact force contains the following elements: Applied force, Air resistance force, Normal force, Spring force, Frictional force, Tension force. The subclass Action - at - a-Distance force contains Gravitational force and Electromagnetic force. The subclass Electromagnetic force contains Electrical force and Magnetic force (see Fig. 10).

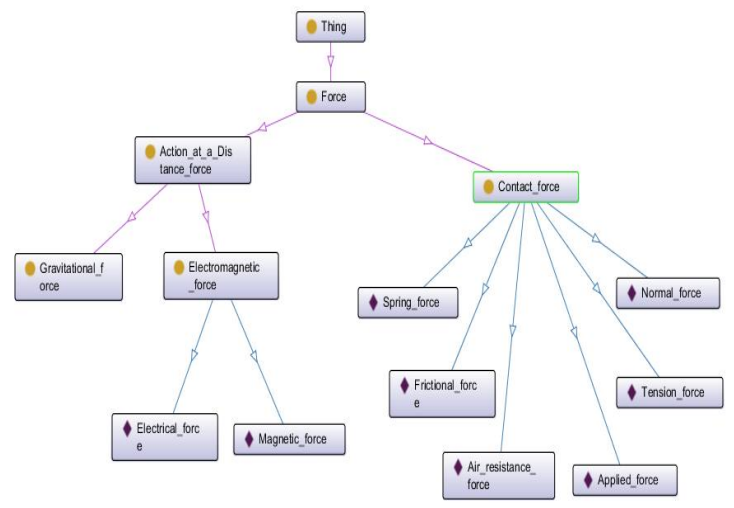

Fig. 10. Visualization of subclasses of the class Force in OntoGrapf tab of Protégé

This example demonstrates that, by using the Protégé, it is possible to create an efficient description of ontologies that may be used for the needs of theoretical mechanics. It can be seen that it is possible to quickly identify the forces that mechanically and directly affect material objects or mechanical systems. Ontology building is a laborious process. For the needs of theoretical mechanics, it is possible to create many coherent ontologies. Due to the fact that the creation of ontologies of theoretical mechanics is in the early stage, the work on building and developing of ontology of theoretical mechanics concepts is to be continued.

\section{CONCLUSION}

Process of development of any ontology is iterative, modification and supplementation is an inevitable part of it. In the future, one of the possible new directions needed for the domain ontology of theoretical mechanics might be related to introduction of new concepts, definition and description of new classes, formation of relations between the concepts and their characteristics.

The results of the study show that, for the forces and their systems studied in theoretical mechanics, it is possible to build various ontologies that would exist and operate simultaneously. The abovementioned examples reveal that, by using ontologies, is possible to demonstrate the links both between material objects and forces, as well as between objects and forces that operate in mechanical systems. It is important to make the newly-created ontologies matching, thus creating a common approach to the needs of theoretical mechanics.

It can be seen that the use of ontology helps us to understand the concepts (forces in this case), their characteristics and existing relations, that create awareness of the forces affecting material objects and their systems, causing their movement or immobility. It provides an efficient solution for the tasks of theoretical mechanics.

The results of this study shall be useful for students while studying theoretical mechanics, they shall also provide understanding of the basic concepts of theoretical mechanics and ontologies.

\section{REFERENCES}

[1] J.S. Walker, Physics. Upper Saddle River, NJ, Pearson / Prentice Hall, 2004.

[2] J.L.Gross and J.Yellen, Graph theory and its applications. Boca Raton, Landon, N.Y. Chapman \& Hall / CRC, 2006, pp. $2-52,534-540$

[3] D. Kleppner and R. Kolenkow, An Introduction to Mechanics. 2nd ed.,Cambridge University Press, 2014, pp. 81 - 110.

[4] V.Solovjov, B.Dobrov, V.Ivanov, and N.Lukashevich, Ontologies and thesauri. Moskwa, 2006. (In Russian)

[5] P. Grabusts, The Concept of Ontology for Numerical Data Clustering, 9th International Conference "Environments. Technology. Resources", Rezekne, Latvia, 2013.

[6] N. Guarino, Formal Ontology in Information Systems, $1^{\text {st }}$ International Conference on Formal Ontology in Information Systems, FOIS, Trento, Italy, IOS Press, pp.3 - 15, 1998

[7] T. R. Gruber, "A Translation Approach to Portable Ontology Specifications", Knowledge Systems Laboratory Computer Science Department Stanford University Stanford, California, Tech. Rep. KSL 92 - 71, 1993.

[8] N.F.Noy and D.L. McGuinness, "Ontology Development 101: A Guide to Creating Your First Ontology", Stanford knowledge systems laboratory, Stanford University, Stanford, California, Tech. Rep. KSL-01-05, 2001. 
[9] V. Devedzic, "Understanding Ontological Engineering," Communications of ACM, vol.45, no.4, pp.136. - 144, April 2002.

[10] A.Gladun and J.Rogushina, "Application of the ontology for enterprises system," Journal "Corporate Systems", No1, 2006. (In Russian)

[11] V. Graudina, "Variety of Definitions and Classifications of Ontologies," Scientific Journal of Riga Technical University, vol.22, pp. 181- 192, 2005.

[12] R. Neches, R. Fikes, T. Finin, T. Gruber, R. Patil, T. Senator, and W. Swartout, "Enabling technology for knowledge sharing," AI Magazine, vol.12, no. 3, pp. 36 - 56, 1991.
[13] N.Guarino and P. Giaretta, "Ontologies and Knowledge Bases Towards a Terminological Clarification", [Online]. Available: http://citeseerx.ist.psu.edu/viewdoc/download?doi=10.1.1.320. 8006\&rep=rep1\&type=pdf [Accessed: Feb.15, 2015].

[14] Protégé project homepage, [Online]. Available: http://protege.stanford.edu/products.php [Accessed: March 30, 2015].

[15] M. Uschold and R. Jasper, "A Framework for Understanding and Classifying Ontology Applications," [Online]. Available: http://www.cs.man.ac.uk/ horrocks/Teaching/cs646/Papers/us chold99.pdf [Accessed: March 3, 2015]. 